\author{
Alcaloides Marinhos Bromopirrólicos \\ Lacerda, R. B.* \\ Rev. Virtual Quim., 2015, 7 (2), 713-729. Data de publicação na Web: 30 de dezembro de 2014 \\ http://www.uff.br/rvq
}

\title{
Bromopyrrole Marine Alkaloids
}

\begin{abstract}
Organic small molecules that are part of the secondary metabolites produced by plants, insects, microorganisms and marine organisms are a rich source of diversity and complexity of unique chemical structures and inspire researchers from different areas of science. Among marine organisms, sponges produce a wide variety of secondary metabolites, many of them having original scaffolds, arousing great interest to medicinal chemistry and pharmacology. These compounds represent an important natural resource in the discovery of new drugs against cancer and other serious diseases. Among them, bromopyrrole alkaloids are part of a family of exclusively marine compounds and are representatives of the chemical diversity of secondary metabolites biosynthesized by marine invertebrates. The interest in this class of natural products has increased due its intriguing and promising pharmacological properties, such as $\alpha$-blockers, serotonin antagonists, kinase activity inhibitors, neuroprotective, antimalarial, analgesic, anticancer, antibacterial, antifungal and antihistamine. Since the discovery of oroidin, the first member of this alkaloids family, hundreds of structurally related compounds have been described, with many different pharmacological activities. Oroidin alkaloids became a relevant source of inspiration for medicinal chemists and has been widely used as prototypes for the design of new chemical entities with pharmacological activity and have already led to several bioactive synthetic analogs described in the literature.
\end{abstract}

Keywords: Marine natural products; bromopyrrole alkaloids; pharmacologic activity; bioactive synthetic analogs.

\section{Resumo}

As micromoléculas orgânicas que fazem parte dos metabólitos secundários produzidos por plantas, insetos, microorganismos e organismos marinhos constituem uma rica fonte de diversidade e complexidade de estruturas químicas originais que servem de inspiração para as pesquisas nas diversas áreas da ciência. Dentre os organismos marinhos, as esponjas produzem uma grande diversidade de metabólitos secundários, muitos dos quais tem estruturas originais de grande interesse para a farmacologia e química medicinal. Esses compostos representam um importante recurso natural, pois podem levar à produção de novos medicamentos contra o câncer e outras doenças graves. Dentre esses compostos, os alcaloides bromopirrólicos fazem parte de uma família de compostos exclusivamente marinhos e são um exemplo da diversidade química dos metabólitos secundários biossintetizados pelos invertebrados marinhos. O grande interesse nesta classe de produtos naturais tem aumentado particularmente devido a suas intrigantes e promissoras propriedades farmacológicas, tais como bloqueadores $\alpha$ adrenérgicos, antagonistas serotoninérgicos, inibidores da atividade quinase, neuroprotetores, antimaláricos, analgésicos, anticâncer, antibacterianos, antifúngicos e anti-histamínicos. Desde a descoberta da oroidina, primeiro membro desta família de alcaloides, centenas de compostos originais estruturalmente relacionados, com diferentes atividades farmacológicas, foram descobertos e descritos na literatura. Esses alcaloides oroidínicos tem sido importante fonte de inspiração para os químicos medicinais e vem sendo amplamente utilizados como protótipos para o planejamento de novas entidades químicas com atividade farmacológica e, já originaram diversos análogos sintéticos bioativos descritos na literatura.

Palavras-chave: Produtos naturais marinhos; alcaloides bromopirrólicos; atividade farmacológica; análogos sintéticos bioativos.

* Universidade Federal Rural do Rio de Janeiro, Instituto de Ciências Exatas, Departamento de Química, SeropédicaRJ, Brasil.

Mrenlacerda@ufrri.br

DOI: $\underline{10.5935 / 1984-6835.20150032}$

Rev. Virtual Quim. |Vol 7| | No. 2| |713-729| 


\title{
Alcaloides Marinhos Bromopirrólicos
}

\author{
Renata B. Lacerda* \\ Universidade Federal Rural do Rio de Janeiro, Instituto de Ciências Exatas, Departamento de \\ Química, Seropédica-RJ, Brasil. \\ * renlacerda@ufrri.br
}

Recebido em 2 de dezembro de 2014. Aceito para publicação em 2 de dezembro de 2014

\section{Produtos Naturais de Origem Marinha}

\section{Os Alcaloides Bromopirrólicos}

2.1. Oroidina

2.2. Dispacamidas

2.3. Nagelamidas

2.4. Himenialdisina

\section{Análogos Sintéticos Bioativos}

\section{Conclusões}

\section{Produtos Naturais de Origem Marinha}

A natureza é a responsável pela produção da maioria das substâncias orgânicas conhecidas, sendo o reino vegetal o principal responsável por grande parte da diversidade e complexidade química conhecida e registrada na literatura até os dias atuais. ${ }^{1} \mathrm{~A}$ variedade de micromoléculas orgânicas que fazem parte dos metabólitos secundários produzidos por plantas, insetos, microorganismos e organismos marinhos é uma consequência de milhões de anos de evolução de diferentes organismos e espécies e, apesar do papel antipredatório ser considerado sua principal função ecológica, estes metabólitos constituem uma rica fonte de diversidade e complexidade de estruturas químicas originais e propriedades farmacológicas que servem de inspiração para as pesquisas nas diversas áreas da ciência. ${ }^{2,3}$

A maioria das moléculas naturais marinhas são produzidas por invertebrados, como esponjas, corais, briozoários e ascídias, e pertencem a diferentes classes estruturais, como poliéteres, terpenos, alcaloides, macrolídeos e polipeptídeos. ${ }^{2,3}$ As esponjas produzem uma grande diversidade de metabólitos secundários, muitos dos quais tem estruturas químicas originais de grande interesse para as pesquisas nas áreas de farmacologia e química medicinal. ${ }^{4,5}$ Esses compostos representam um importante recurso natural, pois podem levar à produção de medicamentos mais eficazes contra o câncer e várias outras doenças graves, como as causadas por vírus, bactérias ou fungos. Dentre os organismos marinhos, as esponjas são um dos grupos com maior percentagem 
de espécies produtoras de compostos com atividades antibióticas, antitumorais e antivirais. $^{5}$

Os alcaloides são reconhecidos pelo seu importante papel na química medicinal devido a seu amplo espectro de atividades biológicas. ${ }^{6}$ Entre os alcaloides de origem marinha, os bromopirrólicos se destacam por constituírem uma família de alcaloides exclusivamente marinhos os quais são amplamente distribuídos em esponjas do mar $^{7}$ pertencentes ao gênero Agelas, Axinella, Acanthella, Pseudoaxinyssa e Hymeniacidon, e representam um importante exemplo da grande variedade de metabólitos secundários formados por esponjas marinhas (Figura 1).

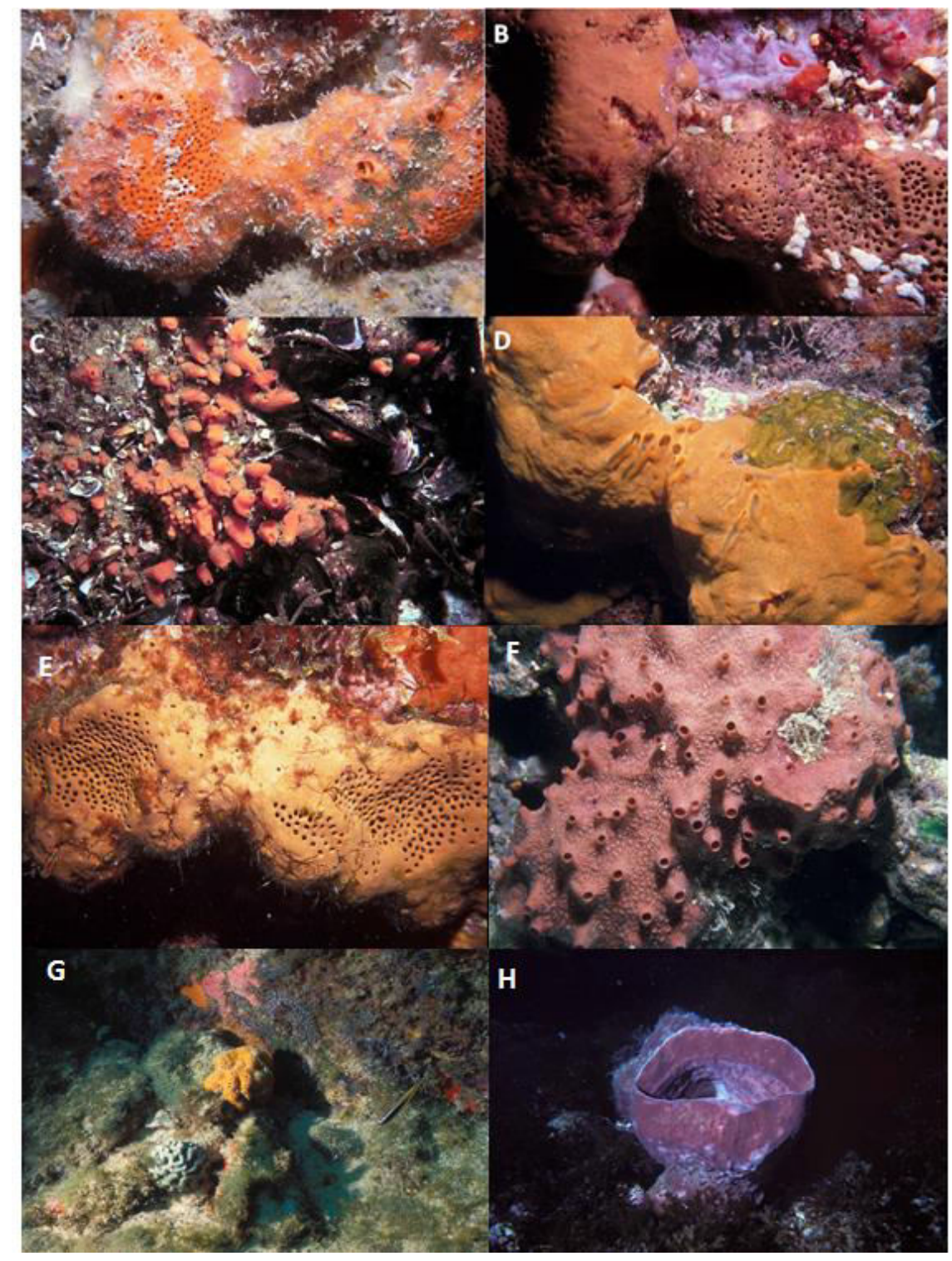

Figura 1. Esponjas marinhas. A- Agelas clathrodes parcialmente recoberta por algas e sedimentos. Fotografada na Bacia Potiguar - RN (Foto F. Moraes, 2007); B- Agelas díspar. Espécime fotografado em lúias ou Pedras Secas (Fernando de Noronha), a 15-18 m de profundidade (Foto G. Muricy, 1990); C- Hymeniacidon heliophila Parker. Fotografia feita no costão do Clube Costa Brava (Rio de Janeiro), a $3 \mathrm{~m}$ de profundidade (Foto G. Muricy); DSpirastrella hartmani fotografada no Atol das Rocas (Foto F. Moraes); E- Agelas díspar (Foto F. Moraes); F- Amphimedon compressa (Foto F. Moraes); G- Axinella corrugata (Foto F. Moraes); H- Xestospongia muta (Foto F. Moraes) 


\section{Os Alcaloides Bromopirrólicos}

Os alcaloides bromopirrólicos fazem parte de uma família de compostos exclusivamente marinhos e são um exemplo da diversidade química dos metabólitos secundários biossintetizados pelos invertebrados do mar. ${ }^{8,9}$ Dentre os organismos marinhos as esponjas do mar são conhecidas por serem a mais rica e importante fonte desta fascinante classe de alcaloides, que se destacam tanto pela sua diversidade e originalidade estrutural como pela riqueza de atividades farmacológicas. ${ }^{10}$

Estes metabólitos secundários são caracterizados estruturalmente pela presença das subunidades mono ou dibromopirrólica, carboxamida e imidazólica funcionalizada, conectadas através de uma cadeia espaçadora alifática, geralmente propílica, funcionalizada ou não. Esses compostos podem existir como monômeros ou dímeros deste quimiotipo, aumentando ainda mais a variabilidade estrutural desta classe de alcaloides. ${ }^{11}$ A maioria dos alcaloides marinhos bromopirrólicos são coletivamente chamados de alcaloides oroidínicos, em referência ao primeiro e mais simples membro representante da classe, a oroidina (1). ${ }^{12,13}$ Dentre os mais de cento e sessenta membros conhecidos desta classe de alcaloides, isolados de mais de vinte esponjas de vários gêneros, podemos citar alguns exemplos como a himenidina (2), ${ }^{14}$ midpacamida (3), ${ }^{15}$ ageladina (4), ${ }^{16}$ as dispacamidas (5A-D), ${ }^{17}$ taurodispacamida $A$ (6) ${ }^{18}$ a 2-bromoceramadina (7), ${ }^{19}$ dispirina $(\mathbf{8})^{20}$ e mukanadinas $\mathrm{E}(\mathbf{9})^{21}$ e G $(\mathbf{1 0})^{19}$ (Figura 2).<smiles>[R]c1[nH]c(C(=O)NC/C=C/c2c[nH]c(N)n2)cc1Br</smiles><smiles></smiles>

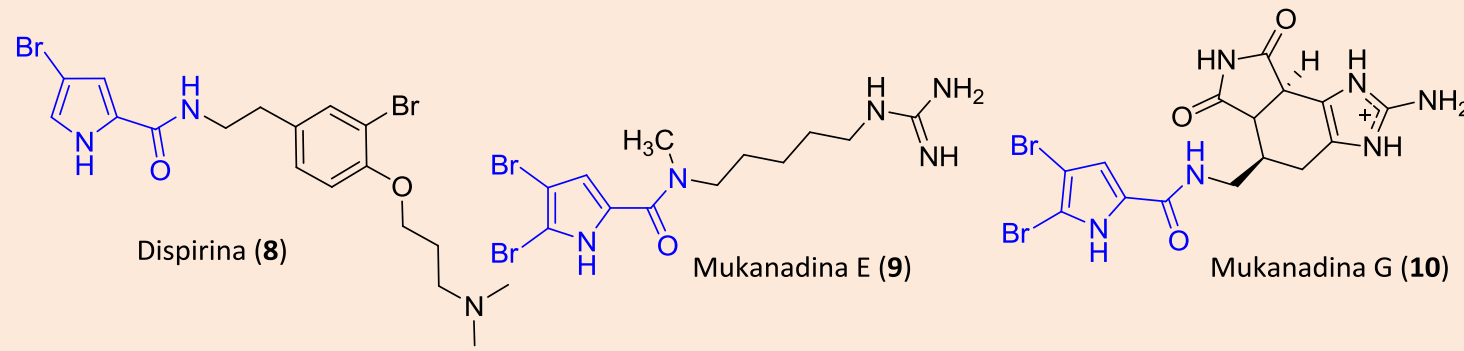

Figura 2. Estruturas químicas de alguns representantes da classe dos alcaloides marinhos oroidínicos 
Do ponto de vista ecológico, o papel antipredatório destes alcaloides pode ser considerado sua principal função biológica, ${ }^{22}$ mas a variedade de atividades farmacológicas apresentada por esta classe de compostos faz dela fonte de investigação como importantes alvos sintéticos visando a caracterização de novos protótipos para estudos químicos, farmacológicos e físico-químicos candidatos a novos fármacos e/ou como ferramentas de uso em biologia celular. ${ }^{23,24}$ Desde o isolamento do primeiro membro deste grupo, a oroidina $(\mathbf{1})^{12,13}$ ao longo dos últimos mais de quarenta anos um expressivo número de moléculas similares e/ou relacionadas foram encontradas, e o grande interesse nesta classe de compostos tem aumentado particularmente devido a suas intrigantes e promissoras atividades farmacológicas, tais como bloqueadores $\alpha$-adrenérgicos, ${ }^{25}$ antagonistas serotoninérgicos, ${ }^{23}$ inibidores da atividade quinase, ${ }^{26}$ neuroprotetores, ${ }^{27}$ antimaláricos, ${ }^{28}$ anticâncer, ${ }^{29}$ antibacterianos, $^{30}$ antifúngicos ${ }^{30}$ e antihistamínicos. ${ }^{17,24}$

\subsection{Oroidina}

A oroidina (1) é o alcaloide marinho bromopirrólico de maior abundância e é considerado como o precursor biogenético dos demais alcaloides desta classe. Como mencionado anteriormente, foi o primeiro membro desta classe de compostos a ser isolado, em 1971 da esponja marinha Agelas oroides, sendo o membro mais simples desta classe de alcaloides marinhos e dando nome a mesma. A oroidina (1) tem uma estrutura linear e aquiral (Figura 3). ${ }^{12,13}$ Ela se caracteriza pela presença do núcleo dibromopirrol carboxamida conectado através de uma cadeia propenílica ao anel imidazólico, sua síntese foi descrita em 2006 e desde então vários análogos sintéticos, como por exemplo inibidores da formação de biofilmes microbianos, vem sendo descritos. ${ }^{31,32}$

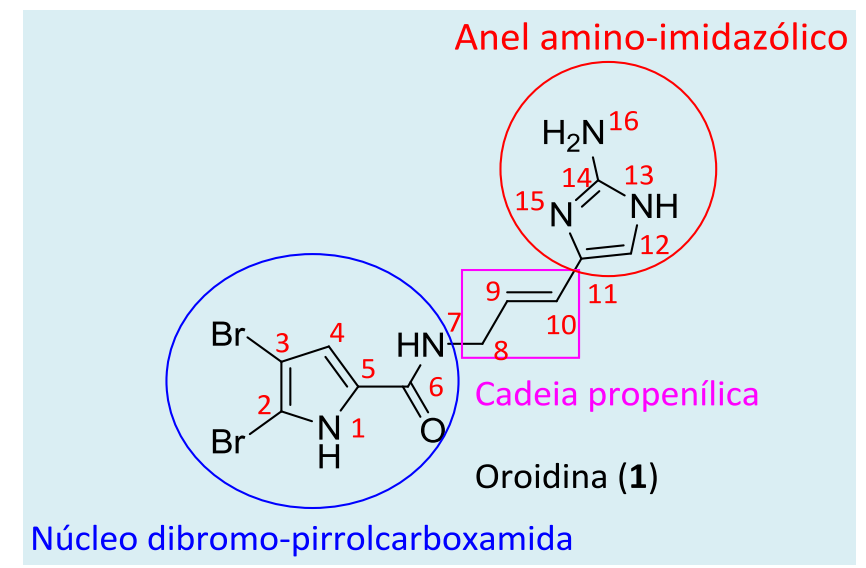

Figura 3. Estrutura química da oroidina (1)

\subsection{Dispacamidas}

As dispacamidas (5A-D) são importantes alcaloides marinhos bromopirrólicos isolados de diversas espécies de Agelas caribenhas ( $A$. conifera, A. longissima, A. clathrodes, A. dispar) e possuem como característica estrutural a presença de uma unidade aminoimidazolônica conectada ao núcleo bromopirrólico através de uma cadeia espaçadora propenílica, que é hidroxilada nas dispacamidas $\mathrm{C}$ e D. ${ }^{33}$ Várias rotas sintéticas para a obtenção das dispacamidas já foram descritas e revisadas na literatura. ${ }^{34,35}$ As dispacamidas (5A-D) tem como característica farmacológica uma atividade antagonista não competitiva na faixa de micromolar sobre os 
receptores histaminérgicos. A atividade antihistamínica das dispacamidas (5A-D) foi observada em modelo in vitro de contração do íleo de cobaia e estes alcaloides se mostraram completamente inativos sobre os receptores colinérgicos e serotoninérgicos, os quais são alvos de outros membros estruturalmente relacionados às dispacamidas (5A-D). ${ }^{17,24}$ A dispacamida B mostrou ainda atividade antiplasmodial contra a cepa multirresistente $\mathrm{K} 1$ de Plasmodium falciparum $\left(\mathrm{IC}_{50}=1,34 \mu \mathrm{M} / \mathrm{ml}\right) .{ }^{36}$

Estruturalmente, a dispacamida (5) se diferencia da oroidina (1) pela isomerização e posição da ligação dupla e, também pela oxidação do anel imidazólico, que se torna uma imidazolona (Figura 4). Diferentemente da oroidina que possui uma ligação dupla com configuração $E$ entre os carbonos C-9 e C-10 em sua cadeia espaçadora propenílica, nas dispacamidas a ligação dupla está entre os carbonos C-10 e C-11 na cadeia espaçadora e tem configuração $Z$ devido ao nitrogênio do anel imidazolônico ser o grupo de maior prioridade na regra CIP (Cahn Ingold Prelog). ${ }^{37} \quad$ Adicionalmente, a cadeia espaçadora das dispacamidas C e D é hidroxilada no carbono $9 .{ }^{24}$
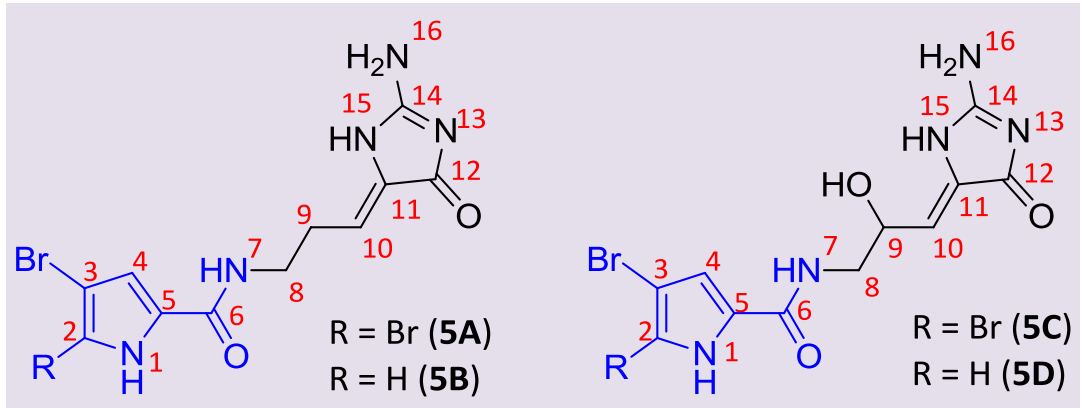

Figura 4. Estruturas química das dispacamidas (5A-D)

\subsection{Nagelamidas}

As nagelamidas são alcaloides bromopirrólicos monoméricos (11U-W) e diméricos acíclicos (11X-Z) isolados de extratos de esponjas marinhas Agelas $s p$.

A nagelamidas $\mathrm{U}$ e $\mathrm{V}$ possuem como características estruturais a presença das subunidades amídica dibromopirrólica, guanidínica e ácido etanossulfônico conectadas a um anel heterociclo pirrolidinona (ou anel $y$-lactâmico). As duas se diferenciam estruturalmente apenas pela configuração do carbono três da pirrolidinona. A nagelamida W se caracteriza pela presença da subunidade amídica dibromopirrólica conectada por um espaçador metilênico a um anel aminoimidazólico que se liga a outro anel idêntico. $^{38}$

As nagelamidas $\mathrm{U}$ e $\mathrm{V}$ são os primeiros exemplos de um alcaloide bromopirrólico contendo o anel $\gamma$-lactâmico e a nagelamida $W$ é o primeiro alcaloide bromopirrólico com duas unidades aminoimidazolônicas na mesma molécula (Figura 5). ${ }^{38}$

As nagelamidas $X$ e $Y$ (Figura 6) são exemplos de alcaloides bromopirrólicos diméricos com um novo esqueleto tricíclico, que consiste das subunidades tetrahidrobenzaminoimidazol e aminoimidazolidina espiro-conectadas. As nagelamidas $X$ e $Y$ são derivadas da oroidina (1) e taurodispacamida A (6) por cicloadição. A nagelamida $Z$ é o primeiro alcaloide bromopirrólico dimérico com dimerização em C- $8 .{ }^{39}$ 
<smiles>[R]C1(NC(=N)N)C[C@H](CNC(=O)c2cc(Br)c(Br)[nH]2)N(CCS(=O)(=O)[O-])C1=O</smiles>

Nagelamidas (11U, $\mathrm{R}=\beta-\mathrm{H}$ e 11V, $\mathrm{R}=\alpha-\mathrm{H})$<smiles></smiles>

Nagelamida (11W)

Figura 5. Estrutura química das nagelamidas (11U-W)

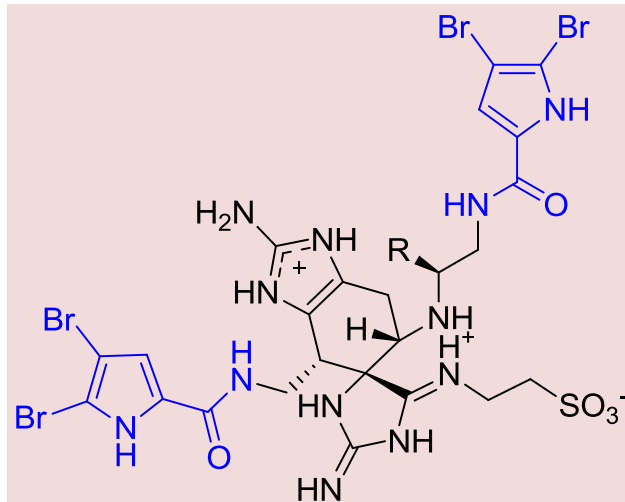

Nagelamidas (11X, R= OH e 11Y, $\mathrm{R}=\mathrm{H}$ )<smiles>Nc1nc(C=CC(NC(=O)c2cc(Br)c(Br)[nH]2)c2[nH]c(N)nc2C=CCNC(=O)c2cc(Br)c(Br)[nH]2)c[nH]1</smiles>

Nagelamida (11Z)

Figura 6. Estrutura química das nagelamidas (11X-Z)

As nagelamidas $U, W, X-Z$ são exemplos de alcaloides marinhos bromopirrólicos com atividade antimicrobiana. As nagelamidas $\mathrm{U}$ e $W$ tem atividade antimicrobiana contra Candida albicans com $\mathrm{IC}_{50}$ de $4 \mu \mathrm{g} / \mathrm{mL}$ e a nagelamida $Z$ apresentou $\quad \mathrm{IC}_{50}$ de $0.25 \mu \mathrm{g} / \mathrm{mL}^{38,39}$

\subsection{Himenialdisina}

A himenialdisina (12) é um monômero cíclico originalmente descrito e caracterizado, em 1982, por difração de raios X. Este alcaloide foi isolado junto com inúmeros metabólitos a partir de uma variedade de esponjas marinhas incluindo espécies Hymeniacidon. Na estrutura de $\mathbf{1 2}$ o espaçador propílico, característico de vários membros da classe, se encontra ciclizado no anel pirrólico formando o núcleo heterociclo tetra-hidropirroloazepinona (Figura 7)..$^{40} \mathrm{~A}$ himenialdisina (12) é descrita na literatura por sua atividade inibitória sobre múltiplas quinases, com atividade na faixa de nanomolar para várias delas, tendo potencial para o tratamento do câncer, doenças inflamatórias e diabetes tipo $2 .^{41} \mathrm{O}$ composto 12 também se mostrou capaz de inibir a fosforilação de várias proteínas neuronais sendo um importante protótipo para o desenvolvimento de novas moléculas para o tratamento de doenças neurodegenerativas como a doença de Alzheimer (DA) e o mal de Parkinson. A himenialdisina (12) é um potente inibidor ATP competitivo de quinase dependente de ciclina 1 (CDK1/ciclina $\mathrm{B} I \mathrm{IC}_{50}=$ $22 \mathrm{nM})$ e 2 (CDK2/ciclina $\left.E, I_{50}=40 \mathrm{nM}\right)$, é um potente inibidor da glicogênio sintase quinase $3 \beta$ (GSK3b, IC ${ }_{50}=10 \mathrm{nM}$ ) o que sugere uma aplicação potencial no tratamento de doenças neurodegenerativas e diabetes. ${ }^{42} \mathrm{O}$ seu análogo não bromado (13), 
outro produto natural marinho, é descrito na literatura por sua boa atividade inibitória sobre as checkpoint kinase 1 e $2,{ }^{1}$ que são serina/treonina quinases envolvidas na regulação do ciclo celular. ${ }^{43}$

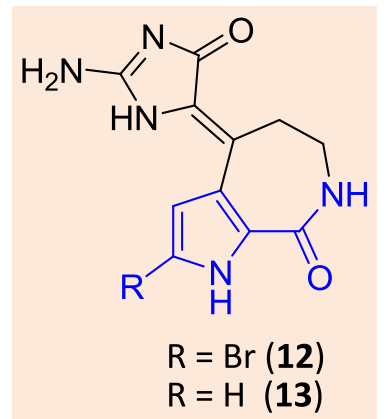

Figura 7. Estrutura química himenialdisina (12) e seu análogo natural não bromado (13)

\section{Análogos Sintéticos Bioativos}

Desde a descoberta da oroidina em 1971, um expressivo número de moléculas similares ou relacionadas aos alcaloides marinhos bromopirrólicos vem sendo descobertas e avaliadas farmacologicamente. $O$ interesse nesta classe de metabólitos secundários de origem marinha vem aumentando principalmente devido a sua grande diversidade de atividades farmacológicas. Estes fatos aliados à crescente necessidade que a Química

1 As checkpoint kinases 1 e 2 são proteínas serina/treonina quinases envolvidas na regulação do ciclo celular, são ativadas quando há um dano no DNA celular, ativando o início dos processos de reparação. Elas limitam a progressão do ciclo celular e previnem a replicação das células até que o DNA danificado seja reparado.

O planejamento de inibidores dessas proteínas quinases constituem uma estratégia para o desenvolvimento de novos fármacos para $\mathrm{o}$ tratamento do câncer.
Medicinal tem de descobrir e/ou inventar novas entidades químicas (NEQ) com atividade farmacológica de interesse terapêutico, tem aumentado significativamente o interesse por esta classe de compostos, que é a mais bem conhecida classe de produtos naturais de origem marinha. Um grande número de análogos sintéticos dos alcaloides marinhos bromopirrólicos vem sendo descritos na literatura, estes compostos são planejados através de várias estratégias de química medicinal, como por exemplo a hibridação molecular ${ }^{44}$ e o bioisosterismo, ${ }^{45}$ para as mais diversas atividades terapêuticas, com destaque para alvos farmacológicos envolvidos no tratamento de câncer, infecções fúngicas e bacterianas, alergias, esquizofrenia, doenças crônicas neurodegenerativas, doenças inflamatórias, etc. Alguns exemplos desses análogos sintéticos e suas respectivas atividades farmacológicas são descritos a seguir.

\section{Agentes antimicrobianos}

As doenças infecciosas constituem um problema de saúde mundial devido ao aumento do número de casos de resistência bacteriana aos fármacos utilizados, o que é um resultado direto do uso crescente desses agentes nas últimas décadas. Adicionalmente, existem também alguns tipos de infecções que ainda não encontram tratamento mesmo com o grande arsenal terapêutico existente nos dias de hoje. ${ }^{46}$ Dessa forma, a busca por novos agentes antimicrobianos capazes de tratar cepas resistentes e outras doenças infecciosas ainda sem tratamento disponível continua sendo um desafio para os químicos medicinais.

Inseridos neste contexto e explorando a diversidade química e farmacológica dos alcaloides bromopirrólicos oroidínicos, Rane e colaboradores descreveram o planejamento e a síntese de vinte novos compostos análogos desta classe de 
metabólitos secundários planejados pela hibridação molecular ${ }^{44}$ da subunidade 4,5dibromopirrólica com a estrutura privilegiada $^{47}$ 1,3,4-oxadiazólica. Os autores estudaram também a substituição dos espaçadores amida e éster, característicos de muitos desses alcaloides, pelo bioisóstero 1,3,4-oxadiazol e investigaram o efeito de vários substituintes no anel sobre a atividade antimicrobiana e antitubercular. Vários análogos sintetizados foram ativos contra Staphylococcus aureus e/ou Escherichia coli como exemplificados aqui pelos compostos híbridos 14-17 (Figura 8). Adicionalmente os resultados também revelaram alguns compostos (16 e 17) como protótipos promissores para o desenvolvimento de novos compostos para o tratamento da tuberculose. $^{48}$<smiles>Clc1ccc(-c2nnc(-c3cc(Br)c(Br)[nH]3)o2)cc1</smiles>

(14)

E. coli $=1,56 \mu \mathrm{g} / \mathrm{mL}$

S. aureus $=1,56 \mu \mathrm{g} / \mathrm{mL}$

atividade antitubecular $=9,6 \mu \mathrm{g} / \mathrm{mL}$<smiles>Fc1ccc(Cl)c(-c2nnc(-c3cc(Br)c(Br)[nH]3)o2)c1</smiles>

(15)

E. coli $=1,56 \mu \mathrm{g} / \mathrm{mL}$

S. aureus $=1,56 \mu \mathrm{g} / \mathrm{mL}$

C. albicans $=1,56 \mu \mathrm{g} / \mathrm{mL}$<smiles>Brc1cc(-c2nnc(-c3ccncc3)o2)[nH]c1Br</smiles>

(16)

S. aureus $=1,56 \mu \mathrm{g} / \mathrm{mL}$ atividade antitubecular $=3,5 \mu \mathrm{g} / \mathrm{mL}$<smiles>Brc1cc(-c2nnc(/C=C/C3=COc4ccccc4C3)o2)[nH]c1Br</smiles>

(17)

S. aureus $=1,56 \mu \mathrm{g} / \mathrm{mL}$

atividade antitubecular $=2,0 \mu \mathrm{g} / \mathrm{mL}$

Figura 8. Compostos híbridos 1,3,4-oxadiazólicos (14-17) análogos sintéticos dos alcaloides marinhos bromopirrólicos com atividade antibacteriana e antitubecular

A hibridação molecular ${ }^{44}$ também foi utilizada por Takale e colaboradores no planejamento de derivados 2-pirrolcarboxamídicos candidatos a agentes antimicrobianos. Nessas moléculas as subunidades 2-pirrol carboxamida e ácido cinâmico foram combinadas através de um espaçador etilênico. Dentre os compostos sintetizados alguns mostraram modesta atividade contra bactérias gram positivas e gram negativas e, podemos destacar o derivado pirrólico carboxamídico não bromado 18 com atividade de $3 \mu \mathrm{g} / \mathrm{mL}$ contra S. aureus (Figura 9). ${ }^{31}$

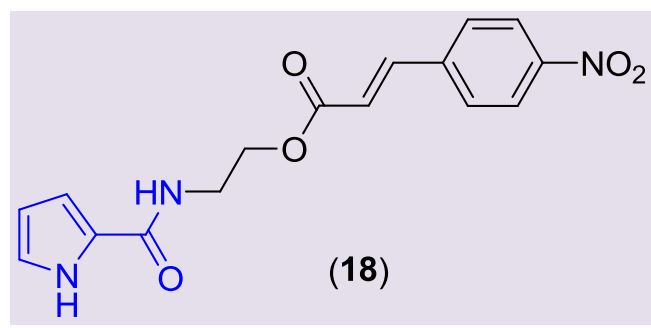

Figura 9. Análogo sintético pirrólico 2-carboxamídico não bromado com atividade antimicrobiana 


\section{Agentes neuroprotetores}

As doenças neurodegenerativas, como por exemplo as doenças de Alzheimer e Parkinson, são importantes fatores de morbidade mundial e representam hoje uma das mais importantes causas de mortalidade no mundo, sendo de grande interesse o desenvolvimento de novos medicamentos com atividade neuroprotetora tanto para a prevenção quanto para o tratamento dessas doenças. ${ }^{49}$

Com o objetivo de descobrir novos inibidores de proteínas quinases terapeuticamente úteis como agentes neuroprotetores, recentemente Guiheneuf e colaboradores descreveram a síntese de novos derivados da rodanina baseados na estrutura do alcaloide marinho bromopirrólico dispacamida A (5). Nos novos análogos sintéticos a subunidade 2aminoimidazolônica característica da dispacamida (5) foi substituída pela subunidade 5-arilidênica da rodanina. Os derivados foram submetidos à triagem farmacológica contra algumas proteínas quinases importantes para 0 desenvolvimento da doença de Alzheimer. Dentre os compostos estudados o derivado 19 (Figura 10), contendo o anel tiooxatiazolidinona, mostrou atividade promissora contra DYRK1A (dual-specificity tyrosine-(Y)-phosphorylation-regulated kinase $1 A)$, uma serina/treonina quinase essencial para o desenvolvimento e função cerebral, com valor de $I_{50}$ de $5,7 \mu \mathrm{M}$ sendo um importante candidato para otimização estrutural, obtenção de compostos mais potentes e estudos de correlações estruturaatividade. ${ }^{50}$

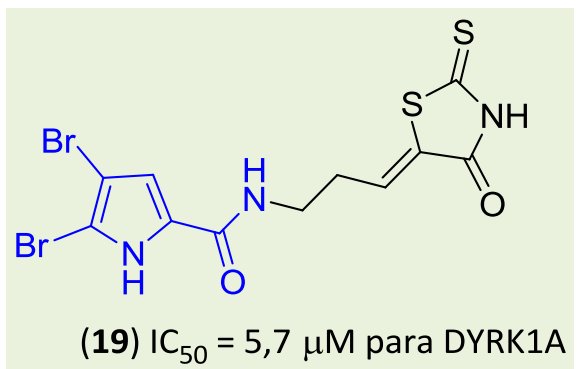

Figura 10. Análogo sintético da dispacamida A (5) com atividade inibitória sobre a DYRK1A

\section{Agentes anti-histamínicos}

A histamina é uma amina biogênica envolvida principalmente em respostas imunes locais e está envolvida em várias doenças humanas como alergias, asma brônquica e úlcera péptica. Estudos também sugerem a participação em doenças do sistema nervoso central como a doença de Alzheimer (DA) e déficit de atenção. A histamina exerce suas ações em quatro tipos diferentes de receptores acoplados a proteína $\mathrm{G}(\mathrm{H} 1-\mathrm{H} 4)$ que são alvos farmacológicos validados para várias doenças e alvos potencias ainda em estudos para várias outras.

Kennedy e colaboradores descreveram a atividade anti-histamínica nos receptores $\mathrm{H} 3$ humanos para $\mathrm{O}$ alcaloide bromopirrólico dispirina (8) $\left(\mathrm{IC}_{50}=2,35 \mu \mathrm{M}\right)^{51}$ e baseados em sua estrutura química sintetizaram uma nova quimioteca de derivados com o objetivo de otimizar sua atividade inibitória além de validar este alcaloide marinho como um novo protótipo para o desenvolvimento de novos antagonistas H3. Os estudos de Kennedy e colaboradores levaram à identificação de dois novos inibidores dos receptores $\mathrm{H} 3$, os análogos sintéticos do alcaloide marinho bromopirrólico dispirina contendo os anéis 
heterociclos de cinco membros oxazol (20) e tiazol (21) (Figura 11) substituindo o núcleo bromopirrólico. Estes análogos bioativos são antagonistas $\mathrm{H} 3$ otimizados em relação ao protótipo natural dispirina (8). ${ }^{52}$<smiles>O=C(NCCc1ccc(OCCN2CCCC2)c(Br)c1)c1cnco1</smiles>

(20) anti-H3 IC $\mathrm{I}_{50}=0,08 \mu \mathrm{M}$<smiles>O=C(NCCc1ccc(OCCN2CCCC2)c(Br)c1)c1nccs1</smiles>

(21) anti-H3 IC $50=0,07 \mu \mathrm{M}$

Figura 11. Análogos sintéticos oxazólico (20) e tiazólico (21) do alcaloide oroidínico dispirina (8) com atividade antagonista $\mathrm{H} 3$ otimizada

\section{Agentes anticâncer}

Apesar do grande avanço alcançado recentemente na descoberta de novos fármacos para o tratamento do câncer, principalmente através da descoberta e validação de novos alvos terapêuticos como as proteínas quinases, o câncer continua sendo a principal causa de morte em todo o mundo. A descoberta de novas micromoléculas capazes de controlar os processos de crescimento e proliferação celular continua sendo um desafio para os pesquisadores principalmente devido a sua natureza multifatorial, desenvolvimento de resistência, toxicidade dos fármacos, etc. E, dentre as fontes de produtos naturais os organismos marinhos são uma importante fonte de metabólitos secundários com atividade antiproliferativa. ${ }^{53}$
Em 2013 Rane e colaboradores descreveram novos análogos sintéticos de alcaloides bromopirrólicos desenhados como compostos híbridos de chalconas, isoxazois e flavonas aos quais foram incorporados a subunidade 4,5-dibromopirrólica. Os autores também investigaram $\mathrm{O}$ efeito da $\mathrm{N}$ metilação da subunidade pirrólica sobre a atividade anticâncer dos novos híbridos, que foram avaliados frente a cinco linhagens de células cancerígenas humanas usando o teste do MTT (redução do 3-(4,5-dimetiltiazol-2-il)2,5-difenil brometo de tetrazolina, um tetrazol amarelo, para formar o formazan, roxo, pelas enzimas mitocondriais das células). Os resultados obtidos pelo grupo mostraram que a introdução do núcleo 4,5dibromopirrol, característico dos alcaloides oroidínicos, nas chalconas, isoxazois e flavonas melhorou o potencial anticâncer dos compostos avaliados, como por exemplo nos compostos híbridos 22-25 (Figura 12). ${ }^{54}$ 
<smiles>[R]n1c(/C=C/C(=O)c2ccccc2O)cc(Br)c1Br</smiles>

(22) $\mathrm{R}=\mathrm{H}$ ou $\mathrm{CH}_{3}$<smiles>[R]n1c(Br)cc(-c2cc(=O)c3ccccc3o2)c1Br</smiles>

(24) $\mathrm{R}=\mathrm{H}$ ou $\mathrm{CH}_{3}$<smiles>COc1ccc(C(=O)/C=C/c2cc(Br)c(Br)n2C)c(OC)c1</smiles><smiles>Cn1c(-c2cc(-c3ccc[nH]3)no2)cc(Br)c1Br</smiles>

(25)

Figura 12. Novos análogos de alcaloides marinhos bromopirrólicos híbridos de chalcona (22 e 23), flavona (24) e isoxazol (25) com atividade anticâncer

A himenialdisina é um importante inibidor de quinases ciclina-dependentes (CDK's) que, entre outras funções, estão envolvidas no controle de ciclo celular e controle transcricional, e das checkpoint kinases 1 e 2 , que também são proteínas quinases reguladoras do ciclo celular. ${ }^{42} \mathrm{~A}$ himenialdisina (12) tem como característica estrutural a presença do farmacóforo heterociclo fundido azepinona e é um importante protótipo para o planejamento de compostos anticâncer. ${ }^{41} \mathrm{O}$ núcleo heterociclo azepinona também está presente na estrutura da kenpaulona (26) (Figura 13), uma indolazepinona sintética capaz de inibir as quinases dependentes de ciclina CDK1/ciclina B $\left(\mathrm{IC}_{50}=0,4 \mu \mathrm{M}\right)$ e CDK2/ciclina A $(I C=0,68 \mu M)$. Assim como a himenialdisina (12), o seu análogo estrutural sintético kenpaulona $(\mathbf{2 6})^{55}$ também vem sendo empregado como protótipo no planejamento de novos inibidores de proteínas quinases. 0 derivado indólico $\mathbf{2 7}$
(Figura 13) é um exemplo de análogo sintético do alcaloide bromopirrólico himenialdisina (12), descrito por Nguyen e colaboradores em 2011, ${ }^{56}$ e é um potente inibidor das checkpoint kinases $1\left(\mathrm{IC}_{50}=220,4\right.$ $\mathrm{nM})$ e $2\left(\mathrm{IC} \mathrm{C}_{50}=13,5 \mathrm{nM}\right)$, que são importantes alvos para o desenvolvimento de fármacos para o tratamento do câncer, ${ }^{43}$ além de inibir a produção de interleucina $2\left({ }^{\prime} C_{50}=3,5 \mu \mathrm{M}\right)$ e TNF $\alpha\left(I_{50}=8.2 \mu \mathrm{M}\right)$, adicionalmente também possui excelente atividade inibitória sobre o crescimento de células $T$ de leucemia $\left(\mathrm{IC}_{50}=1,7 \mu \mathrm{M}\right){ }^{57} \mathrm{~A}$ himenialdisina (12) também foi protótipo para o planejamento da série de derivados hidrazônicos pirrólicos e indólicos descritos por Wan e colaboradores, representados aqui pelo derivado 7-fluoroindólico $28\left(\mathrm{IC}_{50}\right.$ de $\mathbf{0 , 0 8 1}$ $\mu \mathrm{M}$ para $\mathrm{CDK} 5 / \mathrm{p} 25, \mathrm{IC}_{50}$ de $0,171 \mu \mathrm{M}$ para GSK3 $\beta$ e IC $\mathrm{C}_{50}$ de $1,3 \mu \mathrm{M}$ para CDK1/ciclina B), os quais apresentaram atividade antiproliferativa otimizadas em relação ao protótipo. $^{58}$ 
<smiles>O=C1Cc2c([nH]c3ccc(Br)cc23)-c2ccccc2N1</smiles>

Kenpaulona (26)<smiles>NC1=NC(=O)C(=C2CCNC(=O)c3[nH]c4ccccc4c32)N1</smiles>

(27)<smiles>O=C1NCC/C(=N\Nc2ccccn2)c2c1[nH]c1ccc(F)cc21</smiles>

(28)

Figura 13. Estrutura da kenpaulona (26), análogo indólico da himenialdisina $\mathbf{2 7}$ e derivado hidrazônico (28)

Também baseados no farmacóforo tetrahidroazepinona presente em muitos compostos naturais marinhos bioativos, como o alcaloide bromopirrólico himenialdisina (12), White e colaboradores descreveram a síntese e avaliação farmacológica de uma nova série de oximas bromopirrólicas (29) e bromoindólicas (30) (Figura 14). Os compostos descritos por White e colaboradores mostraram atividade antiproliferativa comparável à kenpaulona (26) caracterizando-se como novos candidatos a agentes anti-proliferativos e como protótipos para futura otimização. ${ }^{59}$<smiles>O=C1NCC/C(=N\OCc2ccccc2)c2c1[nH]c(Br)c2Br</smiles>

(29)<smiles>O=C1NCC/C(=N\O)c2c1[nH]c1ccc(Br)cc21</smiles>

(30)

Figura 14. Novas oximas bromopirrólicas (29) e bromoindólicas (30) contendo o núcleo tetraidroazepinona

\section{Conclusões}

Os alcaloides marinhos bromopirrólicos constituem uma das classes de alcaloides com maior diversidade química $\mathrm{e}$ farmacológica conhecida e registrada na literatura. Estes produtos naturais representam um fascinante exemplo da diversidade estrutural de metabólitos secundários produzidos por organismos marinhos e a vasta quantidade de atividades biológicas descritas demonstram uma grande oportunidade para as pesquisas em Química Medicinal. Apesar da surpreendente diversidade estrutural, do expressivo número de compostos bioativos e dos muitos relatos de análogos sintéticos com atividade farmacológica com potencial terapêutico, os alcaloides oroidínicos ainda tem enorme potencial químico e farmacológico a ser explorado para o planejamento e síntese de novos candidatos a protótipos de fármacos. 


\section{Agradecimentos}

Aos Doutores Fernando Coreixas de Moraes e Guilherme Ramos da Silva Muricy (Museu Nacional, Universidade Federal do Rio de Janeiro) por terem gentilmente autorizado a utilização das imagens das esponjas marinhas contidas na Figura 1.

\section{Referências Bibliográficas}

${ }^{1}$ Mishra, B. B.; Tiwari, V. K. Natural products: An evolving role in future drug discovery. European Journal of Medicinal Chemistry 2011, 46, 4769. [CrossRef] [PubMed]

${ }^{2}$ Mayer, A. M. S., Rodriguez, A. D.; Taglialatela-Scafati, O.; Fusetani, N. Marine pharmacology in 2009-11: Marine compounds with antibacterial, antidiabetic, antifungal, anti-inflammatory, antiprotozoal, antituberculosis and antiviral activities; affecting the immune and nervous system, and other miscellaneous mechanisms of action. Marine Drugs 2013, 11, 2510. [CrossRef] [PubMed]

${ }^{3}$ Faulkner, D. J. Highlights of marine natural products chemistry (1972-1999). Natural Products Reports 2000, 17, 1. [CrossRef][PubMed]

${ }^{4}$ Selvin, J.; Lipton, A. P. Biopotentials of secondary metabolites isolated from marine sponges. Hydrobiologia 2004, 513, 231. [CrossRef]

${ }^{5}$ Sipkema, D.; Franssen, M. C. R.; Osinga, R.; Tramper, J.; Wijffels, R. H. Marine sponges as pharmacy. Marine Biotechnology 2005, 7, 142. [CrossRef] [PubMed]

${ }^{6}$ Fattorusso, E.; Taglialatela-Scafati, O.; Modern Alkaloids: Structure, Isolation, Synthesis and Biology, Wiley-VCH, 2008.

${ }^{7}$ Al Mourabit, A.; Potier, P. Sponge's molecular diversity through the ambivalent reactivity of 2-aminoimidazole: A universal chemical pathway to the oroidin-based pyrrole-imidazole alkaloids and their palau'amine congeners. European Journal of Organic Chemistry 2001, 237. [CrossRef]
${ }^{8}$ Faulkner D. J. Marine natural products. Natural Product Reports 2002, 19, 1. [CrossRef] [PubMed]

${ }^{9}$ Faulkner D. J. Marine natural products. Natural Product Reports 2000, 17, 7. [CrossRef] [PubMed]

${ }^{10}$ Rane, R.; Sahu, N,; Shah, C.; Karpoormath, R. Marine bromopyrrole alkaloids: Synthesis and diverse medicinal applications. Current Topics in Medicinal Chemistry 2014, 14, 253. [CrossRef] [PubMed]

${ }^{11}$ Arndt, H.; Riedrich, M. Synthesis of marine alkaloids from the oroidin family. Angewandte Chemie 2008, 47, 4785. [CrossRef] [PubMed]

${ }^{12}$ Forenza, S.; Minale, L.; Riccio, R. New bromo-pyrrole derivatives from sponges agelas-oroides. Chemical Communications 1971, 1129. [CrossRef]

${ }^{13}$ Garcia, E.; Benjamin, L. E.; Frye, R. I. Reinvestigation into the structure of oroidin, a bromopyrrole derivative from marine sponge. Journal of the Chemical Society, Chemical Communications 1973, 78. [CrossRef]

${ }^{14}$ Kobayashi, J.; Ohizumi, Y.; Nakamura, H.; Hirata, Y. A novel antagonist of serotonergic receptors, hymenidin, isolated from the Okinawan marine sponge Hymeniacidon sp. Experientia 1986, 1176. [CrossRef] [PubMed]

${ }^{15}$ Chevolot, L.; Padua, S.; Ravi, B. N.; Blyth, P. C.; Scheuer, P. J. Isolation of 1-methyl-4,5dibromopyrrole-2-carboxylic acid and its 3'(hydantoyl)propylamide (midpacamide) from a marine sponge. Heterocycles 1977, 7, 891. [CrossRef]

${ }^{16}$ Fujita, M.; Nakao, Y.; Matsunaga, S.; Seiki, M.; Itoh, Y.; Yamashita, J.; Van Soest R. W. M.; Fusetani, N. Ageladine A: An antiangiogenic matrixmetalloproteinase inhibitor from the marine sponge Agelas nakamurai. Journal of the American Chemical Society 2003, 125, 15700. [CrossRef] [PubMed]

${ }^{17}$ Cafieri, F.; Fattorusso, E.; Mangoni, A.; Taglialatela-Scafati, $\mathrm{O}$. Dispacamides, antihistamine alkaloids from Caribbean Agelas sponges. Tetrahedron Letters 1996, 37, 3587. [CrossRef] 
${ }^{18}$ Fattorusso, E.; Taglialatela-Scafati, O. Two novel pyrrole-imidazole alkaloids from the Mediterranean sponge Agelas oroides. Tetrahedron Letters 2000, 41, 9917. [CrossRef]

19 Kusama, T.; Tanaka, N.; TakahashiNakaguchi, A.; Gonoi, T.; Fromont, J.; Kobayashi, J. Bromopyrrole alkaloids from a marine sponge Agelas $s p$. Chemical and Pharmaceutical Bulletin 2014, 62, 499. [CrossRef]

${ }^{20}$ Piña, I. C.; White, K. N.; Cabrera, G.; Rivero, E.; Crews, P. Bromopyrrole carboxamide biosynthetic products from the Caribbean sponge Agelas dispar. Journal of Natural Products 2007, 70, 613. [CrossRef] [PubMed]

${ }^{21}$ Yasuda, T.; Araki, A.; Kubota, T.; Ito, J.; Mikami, Y.; Fromont, J.; Kobayashi, J. Bromopyrrole alkaloids from marine sponges of the genus Agelas. Journal of Natural Products 2009, 72, 488. [CrossRef] [PubMed]

${ }^{22}$ Lindel, T.; Hoffmann, H.; Hochgurtel, M.; Pawlik, J. R. Structure-activity relationship of inhibition of fish feeding by sponge-derived and synthetic pyrrole-imidazole alkaloids. Journal of Chemical Ecology 2000, 26, 1477. [CrossRef]

${ }^{23}$ Aiello, A.; D'Esposito, M.; Fattorusso, E.; Menna, M.; Muller, W. E. G.; PerovicOttstadt, S.; Schroder, H. C. Novel bioactive bromopyrrole alkaloids from the Mediterranean sponge Axinella verrucosa. Bioorganic \& Medicinal Chemistry 2006, 14, 17. [CrossRef] [PubMed]

${ }^{24}$ Cafieri, F.; Carnuccio, R.; Fattorusso, E.; TaglialatelaScafati, O.; Vallefuoco, T. Antihistaminic activity of bromopyrrole alkaloids isolated from Caribbean Agelas sponges. Bioorganic \& Medicinal Chemistry Letters 1997, 7, 2283. [CrossRef]

${ }^{25}$ Kobayashi, J.; Nakamura, H.; Ohizumi, Y. Alpha-adrenoceptor blocking action of hymenin, a novel marine alkaloid. Experientia 1988, 44, 86. [CrossRef] [PubMed]

${ }^{26}$ Meijer, L.; Thunnissen, A.; White, A. W.; Garnier, M.; Nikolic, M.; Tsai, L. H.; Walter, J.; Cleverley, K. E.; Salinas, P. C.; Wu, Y. Z.; Biernat, J.; Mandelkow, E. M.; Kim, S. H.; Pettit, G. R. Inhibition of cyclin-dependent kinases, GSK-3 beta and CK1 by hymenialdisine, a marine sponge constituent. Chemistry \& Biology 2000, 7, 51. [CrossRef]

${ }^{27}$ Aiello, A.; D'Esposito, M.; Fattorusso, E.; Menna, M.; Muller, W. E. G.; PerovicOttstadt, S.; Tsuruta, H.; Gulder, T. A. M.; Bringmann, G. Daminin, a bioactive pyrrole alkaloid from the Mediterranean sponge Axinella damicornis. Tetrahedron 2005, 61, 7266. [CrossRef]

${ }^{28}$ Tasdemir, D.; Topaloglu, B.; Perozzo, R.; Brun, R.; O'Neill, R.; Carballeira, N. M.; Zhang, X.; Tonge, P. J.; Linden, A.; Ruedi, P. Marine natural products from the Turkish sponge Agelas oroides that inhibit the enoyl reductases from Plasmodium falciparum, Mycobacterium tuberculosis and Escherichia coli. Bioorganic and Medicinal Chemistry 2007, 15, 6834. [CrossRef] [PubMed]

${ }^{29}$ Xiong, S.; Pang, H.; Fan, J.; Ge, F.; Yang, X.; Liu, Q.; Liao, X.; Xu, S. In vitro and in vivo antineoplastic activity of a novel bromopyrrole and its potential mechanism of action. British Journal of Pharmacology 2010, 159, 909. [CrossRef] [PubMed]

${ }^{30}$ Cafieri, F.; Fattorusso, E.; TaglialatelaScafati, O. Novel bromopyrrole alkaloids from the sponge Agelas dispar. Journal of Natural Products 1998, 61, 122. [CrossRef] [PubMed]

${ }^{31}$ Takale, B. S.; Desai, N. V.; Siddiki, A. A.; Chaudhari, H, K.; Telvekar, V. N. Synthesis and biological evaluation of pyrrole-2carboxamide derivatives: oroidin analogues. Medicinal Chemistry Research 2014, 23, 1387. [CrossRef]

${ }^{32}$ Richards, J. J.; Reyes, S.; Stowe, S. D.; Tucker, A. T.; Ballard, T. E.; Mathies, L. D.; Cavanagh, J.; Melander, C. Amide isosteres of oroidin: Assessment of antibiofilm activity and C. elegans toxicity. Journal of Medicinal Chemistry 2009, 52, 4582. [CrossRef] [PubMed]

${ }^{33}$ Vergne, C.; Appenzeller, J.; Ratinaud, C.; Martin, M.; Debitus, C.; Zaparucha, A.; AlMourabit, A. Debromodispacamides B and D: Isolation from the marine sponge Agelas mauritiana and stereoselective synthesis using a biomimetic proline route. Organic Letters 2008, 10, 493. [CrossRef] [PubMed] ${ }^{34}$ Lindel, T.; Hoffmann, H. Synthesis of dispacamide from the marine sponge Agelas 
dispar. Tetrahedron Letters 1997, 38, 8935. [CrossRef]

${ }^{35}$ Fresneda, P. M.; Molina, P.; Sanz, M. A. A convergent approach to midpacamide and dispacamide pyrrole-imidazole marine alkaloids. Tetrahedron Letters 2001, 42, 851. [CrossRef]

${ }^{36}$ Scala, F.; Fattorusso, E.; Menna, M.; Taglialatela-Scafati, O.; Tierney, M.; Kaiser, M.; Tasdemir, D. Bromopyrrole alkaloids as lead compounds against protozoan parasites. Marine Drugs 2010, 8, 2162. [CrossRef] [PubMed]

${ }^{37}$ Cahn, R. S.; Ingold, C. K.; Prelog, V. Specification of molecular chirality. Angewandte Chemie International Edition 1966, 5, 385. [CrossRef]

${ }^{38}$ Tanaka, N.; Kusama, T.; TakahashiNakaguchi, A.; Gonoi, T.; Fromont, J.; Kobayashi, J. Nagelamides U-W, bromopyrrole alkaloids from a marine sponge Agelas sp. Tetrahedron Letters 2013, 54, 3794. [CrossRef]

${ }^{39}$ Tanaka, N.; Kusama, T.; TakahashiNakaguchi, A.; Gonoi, T.; Fromont, J.; Kobayashi, J. Nagelamides X-Z, dimeric bromopyrrole alkaloids from a marine sponge Agelas sp. Organic Letters 2013, 15, 3262. [CrossRef] [PubMed]

${ }^{40}$ Cimino, G.; De Rosa S.; De Stefanod, S.; Mazzarella, L.; Puliti, R.; Sodano, G. Isolation and X-ray crystal structure of a novel bromocompound from two marine sponges. Tetrahedron Letters 1982, 23, 767. [CrossRef]

${ }^{41}$ Nguyen, T.N.T.; Tepe, J.J. Preparation of hymenialdisine, analogues and their evaluation as kinase inhibitors. Current Medicinal Chemistry 2009, 16, 3122. [CrossRef] [PubMed]

${ }^{42}$ Meijer, L.; Thunnissen, A. M.; White, A. W.; Garnier, M.; Nikolic, M.; Tsai, L. H.; Walter, J.; Cleverley, K. E.; Salinas, P. C.; Wu, Y. Z.; Biernat, J.; Mandelkow, E. M.; Kim, S. H.; Pettit, G. R. Inhibition of cyclin-dependent kinases, GSK-3beta and CK1 by hymenialdisine, a marine sponge constituent. Chemistry and Biology 2000, 7, 51. [CrossRef]
${ }^{43}$ Curman, D.; Cinel, B.; Williams, D. E.; Rundle, N.; Block, W. D.; Goodarzi, A. A.; Hutchins, J. R.; Clarke, P. R.; Zhou, B.-B.; LeesMiller, S. P.; Andersen, R. J.; Roberge, M. Inhibition of the G2 DNA damage checkpoint and of protein kinases Chk1 and Chk2 by the marine sponge alkaloid debromohymenialdisine. The Journal of Biological Chemistry 2001, 276, 17914. [CrossRef] [PubMed]

${ }^{44}$ Viegas-Junior, C.; Danuello, A; da Silva Bolzani, V.; Barreiro, E. J.; Fraga, C. A. Molecular hybridization: a useful tool in the design of new drug prototypes. Current Medicinal Chemistry 2007, 14, 1829. [Crossref] [PubMed]

${ }^{45}$ Lima, L. M.; Barreiro, E. J. Bioisosterism: a useful strategy for molecular modification and drug design. Current Medicinal Chemistry 2005, 12, 23. [Crossref] [PubMed] ${ }^{46}$ Abreu, A. C.; McBainb, A. J.; Simões, M. Plants as sources of new antimicrobials and resistance-modifying agents. Natural Products Reports 2012, 29, 1007. [Crossref] [PubMed]

${ }^{47}$ Duarte, C. D.; Barreiro, E. J.; Fraga, C. A. Privileged structures: a useful concept for the rational design of new lead drug candidates. Mini Reviews in Medicinal Chemistry 2007, 7, 1108. [Crossref] [PubMed]

${ }^{48}$ Rane, R. A.; Gutte, S. D.; Sahu, N. U. Synthesis and evaluation of novel 1,3,4 oxadiazole derivatives of marine bromopyrrole alkaloids as antimicrobial agent. Bioorganic \& Medicinal Chemistry Letters 2012, 22, 6429. [CrossRef] [PubMed]

${ }^{49}$ Dunkel, P.; Chai, C. L. L.; Sperlag, B.; Huleatt, P. B.; Matyus, P. Clinical utility of neuroprotective agents in neurodegenerative diseases: current status of drug development for Alzheimer's, Parkinson's and Huntington's diseases, and amyotrophic lateral sclerosis. Expert Opinion in Investigational Drugs 2012, 21, 1267. [CrossRef] [PubMed]

${ }^{50}$ Guiheneuf, S.; Paquin, L.; Carreaux, F.; Durieu, E.; Roisnel, T.; Meijer, L.; Bazureau, J. New 5-ylidene rhodanine derivatives based on the dispacamide A model. Molecular Diversity 2014, 18, 375. [CrossRef] [PubMed] 
${ }^{51}$ Kennedy, J. P.; Brogan, J. T.; Lindsley, C. W. Total synthesis and biological evaluation of the marine bromopyrrole alkaloid dispyrin: elucidation of discrete molecular targets with therapeutic potential. Journal of Natural Products 2008, 71, 1783. [CrossRef] [PubMed]

52 Kennedy, J. P.; Conn, P. J.; Lindsley, C. W. A novel class of $\mathrm{H} 3$ antagonists derived from the natural product guided synthesis of unnatural analogs of the marine bromopyrrole alkaloid dispyrin. Bioorganic and Medicinal Chemistry 2009, 19, 3204. [CrossRef] [PubMed]

${ }^{53}$ a) Kumar, D.; Rawat, D. S. Marine natural alkaloids as anticancer agents. Opportunity, Challenge and Scope of Natural Products in Medicinal Chemistry 2011, 213; b) Mukherjee, A. K.; Basu, S.; Sarkar, N.; Ghosh, A. C. Advances in cancer therapy with plant based natural products. Current Medicinal Chemistry 2001, 8, 1467. [CrossRef] [PubMed]

${ }^{54}$ Rane, R. A.; Sahu, N. U.; Gutte, S. D.; Mahajan, A. A.; Shah, C. P.; Bangalore, P. Synthesis and evaluation of novel marine bromopyrrole alkaloid based hybrids as anticancer agents. European Journal of Medicinal Chemistry 2013, 63, 793. [CrossRef] [PubMed]
${ }^{55}$ Zaharevitz, D.W.; Gussio, R.; Leost, M.; Senderowicz, A. M.; Lahusen, T.; Kunick, C.; Meijer, L.; Sausville, E. Discovery and initial characterization of the paullones, a novel class of small-molecule inhibitors of cyclindependent kinases. Cancer Research 1999, 59, 2566. [PubMed]

${ }^{56}$ Nguyen, T. N. T.; Saleem, R. S. Z.; Luderer, M. J.; Hovde, S.; Henry, R. W.; Tepe, J. J.; Radioprotection by hymenialdisine-derived checkpoint kinase 2 inhibitors. ACS Chemical Biology 2012, 7, 172. [CrossRef] [PubMed]

${ }^{57}$ Sharma, V.; Lansdell, T. A.; Jin, G.; Tepe, J. T. Inhibition of cytokine production by hymenialdisine derivatives. Journal of Medicinal Chemistry 2004, 47, 3700. [CrossRef] [PubMed]

${ }^{58}$ Wan, Y.; Hur, W.; Cho, C. Y.; Liu, Y.; Adrian, F. J.; Lozach, O.; Bach, S.; Mayer, T.; Fabbro, D.; Meijer, L.; Gray, N. S. Synthesis and target identification of hymenialdisine analogs. Chemistry \& Biology 2004, 11, 247. [CrossRef] [PubMed]

${ }^{59}$ White, A. W.; Carpenter, N.; Lottin, J. R. P.; McClelland, R. A.; Nicholson, R. I. Synthesis and evaluation of novel anti-proliferative pyrroloazepinone and indoloazepinone oximes derived from the marine natural product hymenialdisine. European Journal of Medicinal Chemistry 2012, 56, 246. [CrossRef] [PubMed] 